\title{
ROS-induced oxidative damage in lymphocytes ex vivo/in vitro from healthy individuals and MGUS patients: protection by myricetin bulk and nanoforms
}

\author{
Shabana Akhtar ${ }^{1} \cdot$ Mojgan Najafzadeh ${ }^{1} \cdot$ Mohammad Isreb $^{2} \cdot$ Lisa Newton ${ }^{3} \cdot$ Rajendran C. Gopalan ${ }^{2}$. \\ Diana Anderson ${ }^{1}$ (D)
}

Received: 14 November 2019 / Accepted: 21 February 2020 / Published online: 27 February 2020

(c) The Author(s) 2020

\begin{abstract}
We investigated the protective role of myricetin bulk and nanoforms, against reactive oxygen species (ROS)-induced oxidative stress caused by hydrogen peroxide and tertiary-butyl hydro peroxide in lymphocytes in vitro from healthy individuals and those from pre-cancerous patients suffering with monoclonal gammopathy of undetermined significance (MGUS). The change in intracellular reactive oxygen species was measured once cells were treated with myricetin bulk forms and nanoforms with and without either hydrogen peroxide or tertiary-butyl hydro peroxide co-supplementation. The direct and indirect antioxidant activity of myricetin was spectrofluometrically measured using the fluorescent dye $2^{\prime}, 7^{\prime}$-dichlorofluorescin diacetate and using the Comet assay, respectively. Hydrogen peroxide $(50 \mu \mathrm{M})$ and tertiary-butyl hydro peroxide $(300 \mu \mathrm{M})$ induced a higher level of reactive oxygen species-related DNA damage and strand breaks. Addition of myricetin nanoform $(20 \mu \mathrm{M})$ and bulk $(10 \mu \mathrm{M})$ form could, however, significantly prevent hydrogen peroxide- and tertiary-butyl hydro peroxide-induced oxidative imbalances and the nanoform was more effective. Glutathione levels were also quantified using a non-fluorescent dye. Results suggest that myricetin treatment had no significant effect on the cellular antioxidant enzyme, glutathione. The current study also investigates the effect of myricetin on the induction of double-strand breaks by staining the gamma-H2AX foci immunocytochemically. It was observed that myricetin does not induce double-strand breaks at basal levels rather demonstrated a protective effect.
\end{abstract}

Keywords Myricetin bulk forms and nanoforms $\cdot$ TBHP $\cdot$ hydrogen peroxide $\cdot$ Lymphocytes $\cdot$ MGUS patients $\cdot \gamma$-H2AX

\section{Introduction}

Oxidative stress is a major factor contributing towards the development of various illnesses and it is a key inducer of cellular lesions. It is triggered by a disparity between the production of reactive oxygen species (ROS) and the capacity of biological system to cleanse the reactive intermediates. ROS have been shown to induce proliferation in tumour cells and mediate the proliferation initiated by epidermal growth

Diana Anderson

d.anderson1@bradford.ac.uk

1 School of Chemistry and Biosciences, University of Bradford, Bradford, UK

2 School of Pharmacy and Medical Sciences, University of Bradford, Bradford, UK

3 Bradford Royal Infirmary (BRI), Bradford, UK factor (EGF) or platelet-derived growth factor (PDGF) (Burdick et al. 2003; Chao-Wei Chen et al. 2004). Thus, antioxidants show an effective preventative effect against cancer development by reducing the ROS levels and inhibiting their production. In addition, intracellular ROS production has been associated with the mediation of the effects caused by anti-cancer drugs like taxol (Perkins et al. 2000; Varbiro et al. 2001). Hence data suggests a two-sided role of ROS. It has been suggested that cellular redox potential may be regulated through increasing glutathione (GSH) pools to the mitochondria, hence, preventing ROS formation and cellular injury caused by oxidative stress (Slikker et al. 2001).

Oxidative stress produced by tertiary-butyl hydro peroxide (TBHP) and hydrogen peroxide $\left(\mathrm{H}_{2} \mathrm{O}_{2}\right)$ is well documented. TBHP is a toxic compound and causes extreme discomfort to various organs and systems (Zavodnik et al. 1988; Sarkar and Sil 2010; Bhattacharya et al. 2011). Exposure of cellular components to TBHP results in an increase 
of membrane permeability along with hyperpolarization. Reaction of TBHP with haemoglobin forms t-butoxyl radicals, which then initiate peroxidation by interacting with membrane lipids (Deuticke et al. 1987). Extensive lipid peroxidation leads to membrane disturbance (Benatti et al. 1982). Cellular antioxidant enzymes, like glutathione and 1-ascorbate, to some level inhibit membrane disruption by scavenging the t-butoxyl radicals.

Myricetin (3,5,7,3', 4', 5'-hexahydroxyflavone), an important flavonol, is widely found in berries, fruit, and vegetables (Wang et al. 2010). A large body of data has been published regarding the antioxidant potential of myricetin, leaving no doubt that the compound is a strong antioxidant. It depicts antioxidant activity by scavenging ROS through oxidation of the three hydroxyl groups $\left(3^{\prime}, 4^{\prime}, 5^{\prime}\right.$-position) attached in its B ring (Chobot and Hadacek 2011). Substantial amounts of evidence suggest that myricetin shows other promising health beneficiary effects including chemo preventative, anti-diabetic, anti-cancer, anti-inflammatory, anti-allergic, and activity against cardiovascular diseases (Semwal et al. 2016). Studies have demonstrated that the physiological concentrations range of myricetin $(5-10 \mu \mathrm{M})$ (Peng and Kuo 2003) could significantly inhibit the production of peroxynitrite-induced double-strand breaks (DSBs) (Chen et al. 2011) and protect against the oxidative damage caused in neurodegenerative disorders (Laabich et al. 2007; Shimmyo et al. 2008). Myricetin has been demonstrated to prevent the TBHP-induced chemiluminescence of mouse liver homogenates (Fraga et al. 1987) and oxidative stress in erythrocytes from Type-2 diabetic patients in vitro (Pandey et al. 2009). $\mathrm{H}_{2} \mathrm{O}_{2}$-induced oxidative stress and DNA strand breaks caused in human lymphocytes and human colonocyte cells have been demonstrated and shown to be reduced upon myricetin treatment (Dutie et al. 1997).

Although myricetin is a well-established antioxidant, little is known about its beneficial role in countering the TBHP-induced cytotoxicity in primary lymphocytes ex vivo in vitro of healthy individuals and monoclonal gammopathy of undermined significance (MGUS) patients. MGUS is an asymptomatic precursor (pre-cancerous) condition of multiple myeloma (MM) and it is well documented that almost every case of MM is headed by MGUS (Dhodapkar 2016). The aim of the present study was, therefore, to investigate the in vitro protective role of myricetin nanoparticles (MYR N) and myricetin bulk (MYR B) against TBHP-induced oxidative damage in lymphocytes from healthy individuals and MGUS patients. The effect of myricetin nanoforms and bulk forms against $\mathrm{H}_{2} \mathrm{O}_{2}$-induced damage, change in intracellular ROS due to oxidative stress caused by TBHP and change in GSH levels was investigated. The study also assessed the effect of myricetin nanoforms and bulk forms on DSBs at basal levels by analysing the gamma-H2AX protein expression immunocytochemically.

\section{Methodology}

\section{Blood sample collection and ethics}

The current project involving the use of human peripheral lymphocytes has been granted ethical approval by Leeds East Ethics Committee (IRAS Reference No.:12/ YH/0464) and the University of Bradford's Sub-Committee for Ethics in Research involving healthy Human Subjects (Reference No.: 0405/8). All peripheral blood samples were collected after informed consent from patients and healthy individuals. The research support and governance office of Bradford Teaching Hospitals NHS Foundation also agreed the research (REDA number 1202).

\section{Informed consent}

The blood samples from healthy individuals and MGUS patients used in the current study were collected after obtaining informed consent from the participants and are listed in Tables 1 and 2, respectively. Exclusion criteria for human subjects enrolled in the study were anaemia, blood diseases or major disorders.

Table 1 Healthy blood samples

\begin{tabular}{llllll}
\hline No & Age & Ethnicity & Gender & $\begin{array}{l}\text { Smoking } \\
\text { history }\end{array}$ & Family history \\
\hline 1 & 48 & Caucasian & M & No & None \\
2 & 28 & Caucasian & M & No & None \\
3 & 27 & African & M & Yes & None \\
4 & 38 & Caucasian & M & No & None \\
5 & 60 & Caucasian & F & No & None \\
6 & 40 & Arab & M & No & None \\
7 & 45 & Caucasian & M & Yes & None \\
8 & 55 & Caucasian & M & No & None \\
9 & 35 & Caucasian & M & Yes & None \\
10 & 25 & Caucasian & M & No & None \\
11 & 44 & Asian & M & Yes & None \\
12 & 28 & Caucasian & M & No & None \\
13 & 23 & Caucasian & F & No & none \\
14 & 27 & Caucasian & M & No & None \\
15 & 33 & Arab & M & Yes & None \\
16 & 47 & Asian & M & Yes & None \\
17 & 28 & Caucasian & M & No & None \\
18 & 42 & Asian & M & No & None \\
19 & 48 & Asian & M & No & None \\
20 & 60 & Asian & M & Yes & None \\
\hline & & & & & \\
\hline
\end{tabular}


Table 2 Brief information about patient samples

\begin{tabular}{lllllll}
\hline No & Age & Ethnicity & Gender & $\begin{array}{l}\text { Smoking } \\
\text { history }\end{array}$ & Family history & Medical condition \\
\hline 1 & 79 & Caucasian & M & No & None & MGUS \\
2 & 80 & Caucasian & F & No & None & MGUS \\
3 & 78 & Caucasian & M & No & None & MGUS \\
4 & 56 & Caucasian & F & Yes & Leukaemia and brain Tumour & MGUS \\
5 & 77 & Caucasian & M & No & None & MGUS \\
6 & 75 & Caucasian & M & No & None & MGUS \\
7 & 80 & Caucasian & M & No & Cancer Positive & MGUS \\
8 & 81 & Caucasian & F & No & Bowel and stomach & MGUS \\
9 & 63 & Caucasian & M & Yes & None & MGUS \\
10 & 55 & Caucasian & M & No & None & MGUS \\
11 & 83 & Caucasian & M & No & None & MGUS \\
12 & 63 & Caucasian & M & Yes & None & MGUS \\
13 & 74 & Caucasian & M & No & None & MGUS \\
14 & 63 & caucasian & F & Yes & Arthritis & MGUS \\
15 & 66 & Caucasian & F & No & Breast cancer & MGUS \\
16 & 52 & caucasian & M & Yes & None & MGUS \\
17 & 83 & Caucasian & M & No & None & MGUS \\
18 & 60 & Asian & F & No & None & MGUS \\
19 & 75 & Caucasian & F & No & Mastectomy & \\
20 & 69 & Caucasian & M & No & Stomach and lung & \\
\hline
\end{tabular}

$M$ male, $F$ female, $M G U S$ monoclonal gammopathy of unknown significance

\section{Preparation of myricetin bulk forms and nanoforms, concentration, solubility, and zeta potential of nanoparticles}

Myricetin powder (' $96 \%$ purity) was purchased from Fisher Scientific, UK. Suspensions of myricetin bulk forms and nanoforms were made in an excipient mixture (containing 7\% $(\mathrm{w} / \mathrm{w})$ solid loads of myricetin in a medium comprising of hydroxypropyl methylcellulose (HPMC) $(0.5 \% \mathrm{w} / \mathrm{w})$, sodium lauryl sulphate (SLS) $(0.1 \% \mathrm{w} / \mathrm{w})$, ethanol $(0.8 \% \mathrm{w} / \mathrm{w})$, polyvinylpyrrolidone (PVP) K-30 $(0.5 \% \mathrm{w} / \mathrm{w})$ and purified water). The suspensions were transferred to an amber glass bottles and stored at $4{ }^{\circ} \mathrm{C}$ for the research duration. The mean particle sizes and zeta potential (ZP) of myricetin bulk and nano in the stock solutions were measured using a Zetasizer Nano ZS-90 Model ZEN3600 (Malvern Instruments Ltd, UK) by photon correlation spectroscopy. The initial particle sizes of bulk and nano were 1737 and $161 \mathrm{~nm}$, respectively and their ZP measurement was less than $1 \%$ indicating their good stability. The suspensions were also sonicated for $10 \mathrm{~min}$ before each use to avoid sedimentation and control aggregation.

\section{In vitro experimental design, preliminary treatment, and concentration range study in lymphocytes}

TBHP is a known intracellular stress inducer hence; increase in ROS production was used as a factor to choose the optimum concentration of TBHP for $0-60 \mathrm{~min}$. The concentration dependent responses of TBHP are shown in Fig. 1a. TBHP has induced significant levels of ROS at each determined concentration after 60 min of treatment. However, at $300 \mu \mathrm{M}$, its activity was at highest when compared to the untreated group.

Flavonoid compounds have this ability to cause adverse effects at slightly different concentrations; therefore, we used the safer concentrations with no significant genotoxicity. Based on the dose-response studies, $10 \mu \mathrm{M}$ for MYR B and $20 \mu \mathrm{M}$ for MYR N were optimal doses with no cellular toxicity (Fig. 1b).

The optimal concentration of $\mathrm{H}_{2} \mathrm{O}_{2}(50 \mu \mathrm{M})$ used throughout the study as the positive control and for co-supplementation in the Comet assay was determined by the dose response 

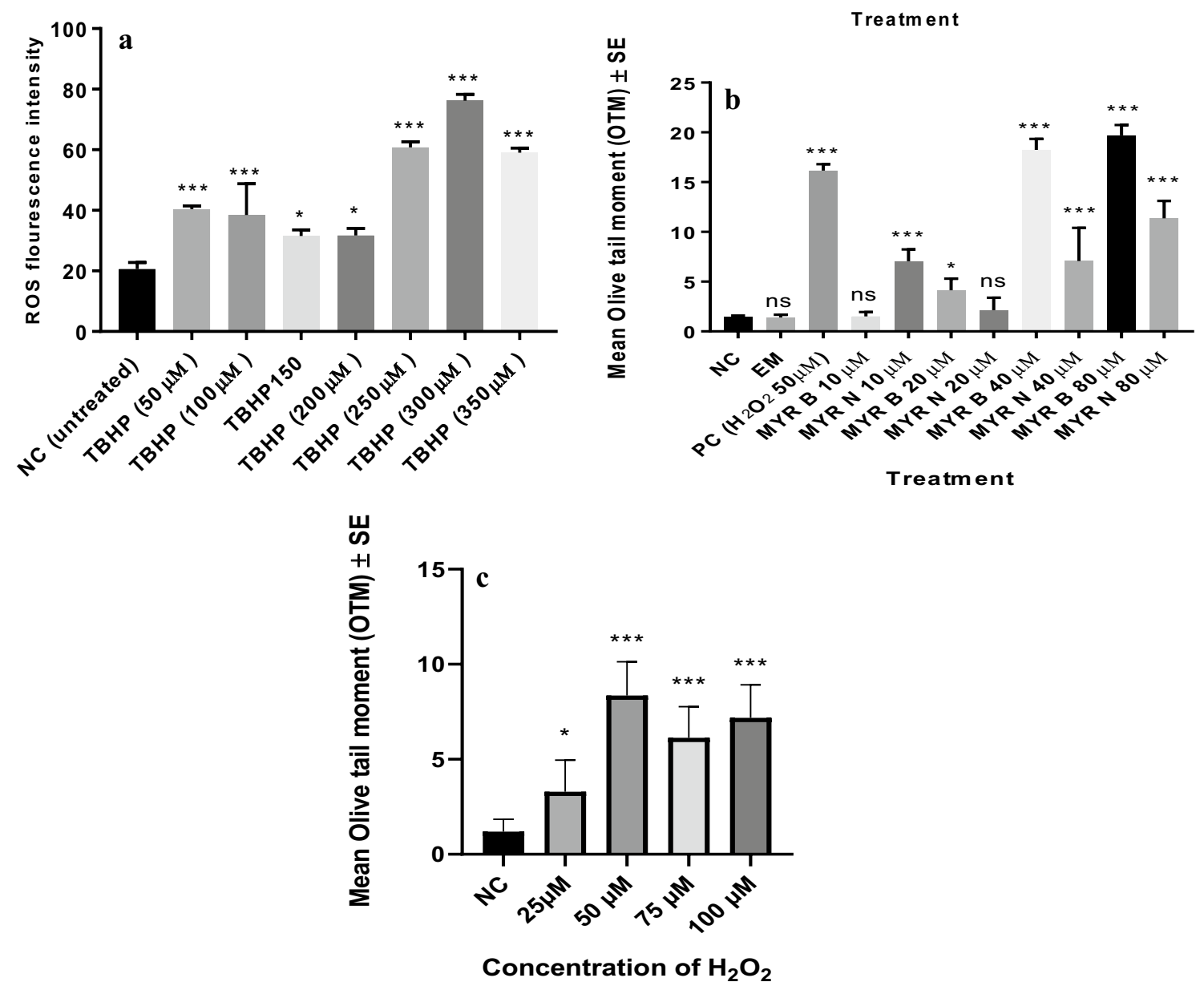

Fig. 1 a Average of three independent experiments showing, change in intracellular ROS after treatment with different concentrations of TBHP in healthy lymphocytes. All groups were compared against the untreated group. $* * * P<0.001, * P=0.02$. Results are expressed as mean \pm SE. b Concentration dependent responses of MYR B and MYR $\mathrm{N}$ in healthy lymphocytes showing mean OTM. All data have been expressed as mean \pm standard errors (SE) of experiments in healthy individuals, measuring 100 cells each. In vitro treatment of healthy lymphocytes with different concentrations of MYR B and MYR N in the Comet assay has shown that MYR B $(10 \mu \mathrm{M})$ and MYR N $(20 \mu \mathrm{M})$ produced least levels of the DNA damage which was comparative with the negative control (NC). Therefore, these optimum concentrations have been used throughout the study. Other

(1c). Untreated lymphocytes were set as the negative control (NC). The optimal concentrations for TBHP and $\mathrm{H}_{2} \mathrm{O}_{2}$ were used as positive controls (PC). After determining the optimum doses for the test chemicals, PCs were simultaneously added to lymphocytes in suspension with either MYR B or MYR N in co-supplementation studies.

\section{Confounding factors}

The potential effects of the confounder such as smoking, age, ethnicity, and gender on results were analysed using treatment groups included, excipient mixture (EM) (0.1\%) and PC $\left(\mathrm{H}_{2} \mathrm{O}_{2} 50 \mu \mathrm{M}\right)$. The stars indicate significant difference between $\mathrm{NC}$ and various treatment groups $(P<0.05$ significantly different $), n s$ indicates not significant, $* * * P<0.001, * P<0.01$. c Concentrationdependent responses of $\mathrm{H}_{2} \mathrm{O}_{2}$ in healthy lymphocytes showing mean OTM. All data have been expressed as mean \pm standard errors (SE) of experiments in healthy individuals, measuring 100 cells each. In vitro treatment of healthy lymphocytes with different concentrations of $\mathrm{H}_{2} \mathrm{O}_{2}$ in the Comet assay has shown that $50 \mu \mathrm{M}$ of $\mathrm{H}_{2} \mathrm{O}_{2}$ produced most significant levels of the DNA damage compared to the NC. Therefore, this optimum concentration has been used throughout the study as a PC $(P<0.05$ was considered significantly different $), n s$ indicates not significant, ${ }^{* * *} P<0.001,{ }^{*} P<0.01$

the Comet assay. However, myricetin had no significantly different effect on any of these factors (Table 3 ).

\section{MTT assay}

The cytotoxicity of chemicals was determined by 3-(4,5-dimethylthiazol-2-yl)-2,5-diphenyltetrazolium bromide (MTT) dye absorbance. Isolated lymphocytes $\left(10^{4}\right)$ were maintained in complete medium overnight in 96-well plates followed by treatment the next day. After the exposure time ( 1 and $24 \mathrm{~h}$ ) was over, $10 \mu \mathrm{l}$ of MTT dye (Fisher 
Table 3 The effect of confounding factors in healthy individuals and MGUS patients using OTM

\begin{tabular}{lllllllrrr}
\hline Subject & Treatment group & $\begin{array}{l}\text { Smoker } \\
\text { OTM }\end{array}$ & $\begin{array}{l}\text { Non-smoker } \\
\text { OTM }\end{array}$ & $\begin{array}{l}\text { Asian } \\
\text { OTM }\end{array}$ & $\begin{array}{l}\text { Caucasian } \\
\text { OTM }\end{array}$ & $\begin{array}{l}\text { Male } \\
\text { OTM }\end{array}$ & $\begin{array}{l}\text { Female } \\
\text { OTM }\end{array}$ & $\begin{array}{l}\text { Young } \\
\text { OTM }\end{array}$ & $\begin{array}{l}\text { Old } \\
\text { OTM }\end{array}$ \\
\hline Healthy individuals & NC & 1.1 & 0.7 & 0.7 & 0.7 & 1.2 & 0.8 & 2.7 \\
& PC $\left(\mathrm{H}_{2} \mathrm{O}_{2} 50 \mu \mathrm{M}\right)$ & 8.9 & 10.7 & 11.8 & 8.9 & 8.1 & 13.5 & 6.7 & 10.3 \\
& MYR B $(10 \mu \mathrm{M})$ & 2.0 & 1.49 & 1.1 & 2.0 & 1.5 & 1.3 & 3.1 & 6.0 \\
& MYR N $(20 \mu \mathrm{M})$ & 1.5 & 0.8 & 1.4 & 0.89 & 2.0 & 1.2 & 1.6 & 3.05 \\
MGUS patients & $\mathrm{NC}$ & 3.6 & 2.8 & 4.0 & 2.5 & 2.2 & 2.6 & 3.5 & 3.2 \\
& PC $\left(\mathrm{H}_{2} \mathrm{O}_{2} 50 \mu \mathrm{M}\right)$ & 9.0 & 8.7 & 15.5 & 6.2 & 6.4 & 5.4 & 9.5 & 11.9 \\
& MYR B $(10 \mu \mathrm{M})$ & 2.5 & 3.4 & 6.9 & 3.8 & 2.6 & 3.3 & 3.9462 & 4.5 \\
& MYR N $(20 \mu \mathrm{M})$ & 2.3 & 1.7 & 4 & 2.9 & 1.0 & 1.8 & 2.1384 & 3.1 \\
\hline
\end{tabular}

Scientific, UK) (5 mg/ml) was added to each well and incubated for another $4 \mathrm{~h}$ at $37^{\circ} \mathrm{C}$ in the dark. After this, medium was aspirated and $200 \mu \mathrm{l}$ of DMSO (Fisher Scientific, UK) was added to each treatment and absorbance was read at $570 \mathrm{~nm}$.

\section{The Comet assay for determination of DNA damage}

Lymphocytes were treated with MYR B $(10 \mu \mathrm{M})$ and MYR $\mathrm{N}(20 \mu \mathrm{M})$ in combination with $\mathrm{H}_{2} \mathrm{O}_{2}(50 \mu \mathrm{M})$ for $1 \mathrm{~h}$. The cell suspension was centrifuged at $3000 \mathrm{rpm}$ (1000 g). The supernatant was removed and the pelleted cells were subjected for the Comet assay as previously defined with some changes (Singh et al. 1988; Tice et al. 2000; OECD 2016; Anderson et al. 2014; Azqueta and Dusinska 2015).

\section{Measurement of ROS accumulation in isolated lymphocytes}

Isolated lymphocytes were grown in a 96-well plate overnight. Cells were washed with phosphate buffer saline (PBS) prior to addition of various chemical treatments in PBS and incubated for $1 \mathrm{~h}$ at $37^{\circ} \mathrm{C}$ in the presence of $5 \% \mathrm{CO}_{2}$. Once incubation was over, the cells were washed again with PBS following the loading of a fluorescent probe, DCFDA dye (Abcam, UK) into each well and incubated again for $45 \mathrm{~min}$ under the same conditions. Then dye was replaced by $100 \mu \mathrm{l}$ of PBS and fluorescence was measured at 485/535 nm using Promega Glomax explorer version 2.4. Data were analysed using the Graph Pad Prism 7.02.

\section{Assay of cellular enzyme and total thiol content}

Reduced glutathione (GSH) levels and oxidised glutathione (GSSG) contents in experimental and normal lymphocytes were analysed using the GSH/GSSG Ratio Detection Assay Kit (Fluorometric-Green) (Abcam, UK) according to the manufacturer protocol. Briefly, isolated lymphocytes were harvested overnight in 6-well plate then treated with chemicals for $1 \mathrm{~h}$. Cells were washed with cold PBS and resuspended in $100 \mu \mathrm{l}$ of cold lysis buffer supplemented with $10 \mu$ l of protease inhibitors then thoroughly mixed by pipetting and centrifuged at $400 \mathrm{~g}$ for $5 \mathrm{~min}$ to remove insoluble. The supernatant (sample) was collected and kept on ice for further use. $50 \mu \mathrm{l}$ of each GSH and GSSG standards (kit components) were added to 96-well plates in duplicates. For GSH detection, $50 \mu \mathrm{l}$ of the GSH assay mixture (GAM) (provided with the kit) was added to each GSH standard and sample. For GSSG detection, total GSH assay mixture (TGAM) was added to each GSSG standard and sample. Incubated for $60 \mathrm{~min}$ at room temperature in the dark and then fluorescence was measured at 490/520 nm using Promega Glumax explorer version 2.4. Data were analysed using Graph Pad Prism 7.02.

\section{Determination of histone-2AX (H2AX) phosphorylation ( $\gamma$-H2AX) levels using Immunocytochemistry}

The method was based on that of Schmid et al. (2010) and previously followed in our lab (Laubenthal et al. 2012). A total of 100 cells per treatment were examined under the fluorescence microscope connected with a CCD camera (Nikon Digital Sight DS-SMC, Surrey, UK) for gammaH2AX foci expression, a marker of DNA double-strand breaks. Untreated cells were used as the negative control, whereas doxorubicin $(50 \mu \mathrm{M})$ was used as the positive control. Each experiment was done in triplicate with both controls included.

\section{Statistical analysis}

Results were presented as mean $\pm \mathrm{SE}$ and differences between the groups were analysed by one-way and two-way analysis of variance (ANOVA) using Graph Pad prism 7 software. Values of $p<0.05$ were considered significantly different. 


\section{Results}

\section{Cytotoxicity of chemicals in lymphocytes}

Cytotoxicity of test chemicals was determined by culturing lymphocytes from healthy individuals and MGUS patients and measuring the mean absorbance at 1 and $24 \mathrm{~h}$ using the MTT dye. Results from the MTT assay showed that the test chemicals used in this study exhibited non-significant level of cytotoxicity (which was approximately 5\% lower compared to the untreated cells) occurred only after $24 \mathrm{~h}$ treatment, for all the treatment groups assessed in lymphocytes from both healthy and patient group (Fig. 2a, b).
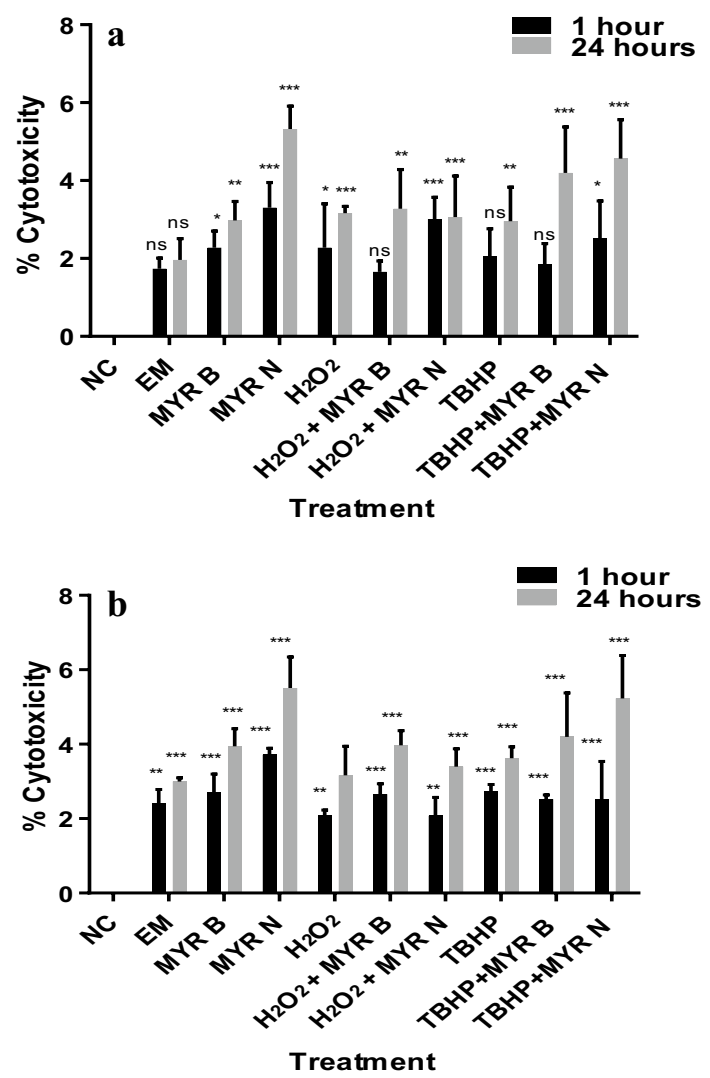

Fig. $2 \%$ Cytotoxicity in $2 \times 10^{4}$ cells of various test treatments in lymphocytes from healthy volunteers (a) and MGUS patients (b) using MTT assay. Overall values were less than $5 \%$ which is not considered as cytotoxic. Error bars show mean values $\pm \mathrm{SE}, n=3$. $* P=0.03, * * P=0.002, * * * P=0.0002, n s$ not significant
Effect of MYR N \& B on hydrogen peroxide $\left(\mathrm{H}_{2} \mathrm{O}_{2}\right)$-induced DNA damage in lymphocytes from healthy vs patient group using the Comet assay

In-vitro treatment of lymphocytes from healthy individuals and patients with MYR B $(10 \mu \mathrm{M})$ and MYR N $(20 \mu \mathrm{M})$, co-supplemented with $50 \mu \mathrm{M} \mathrm{H}_{2} \mathrm{O}_{2}$, resulted in significant reduction of DNA damage when compared to the PC $\left(50 \mu \mathrm{M} \mathrm{H}_{2} \mathrm{O}_{2}\right)$ alone. MYR N and $\mathrm{NC}$ show the same level of DNA damage in healthy lymphocytes both in \%tail DNA and Olive tail moment (OTM). The $\mathrm{H}_{2} \mathrm{O}_{2}$-induced DNA damage was significantly reduced by MYR B and MYR N in both groups, whereas MYR N was more effective in healthy group when compared to the PC (Fig. 3a, b), giving the value almost similar to the untreated group.

\section{Antioxidant effects of myricetin}

The study used a cell permeant reagent $2^{\prime}, 7^{\prime}$-dichlorofluorescin diacetate (DCFDA) that measures total intracellular ROS activity and levels. Once diffused into the cells, cellular esterases deacetylate DCFDA as a non-fluorescent form which is later oxidized to a highly fluorescent compound, 2', 7'-dichlorofluorescein (DCF), by interacting with ROS and easily detected by fluorescence spectroscopy. The effect of MYR B and MYR N on basal ROS was determined in lymphocytes from the healthy group and pre-cancerous patients. Results showed that MYR N has significantly reduced the ROS levels in lymphocytes from both groups.

\section{TBHP-induced oxidative injury in lymphocytes: protection by myricetin}

Exposure of healthy and patient lymphocytes to TBHP alone induced a significant increase in ROS levels by $40 \%$. However, upon treatment with MYR B and MYR N, a significant attenuation of TBHP-induced ROS was observed in lymphocytes from both groups (Fig. 4a, b).

\section{Activity of intracellular antioxidant enzyme, GSH, and change in GSH/GSSG ratio}

The effects of MYR B and MYR N on the basal levels of GSH were assessed by treating lymphocytes from healthy and patient group with both forms of myricetin alone. MYR B and MYR N have shown a trend to increase the basal levels of GSH/GSSG ratio in lymphocytes from precancerous patients, but levels are not significantly different. Hence, myricetin does not show any substantial effect 


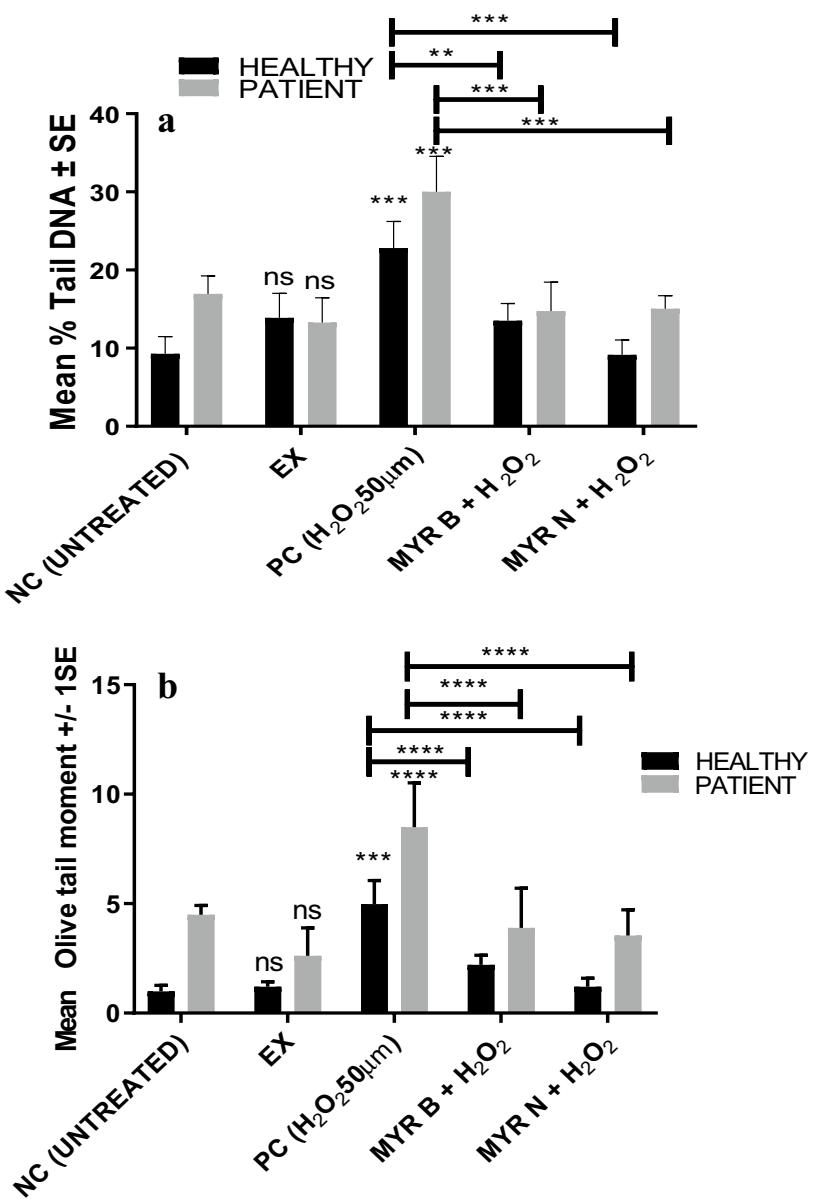

Fig. 3 a Mean \% tail DNA showing effect of MYR N \& B on $\mathrm{H}_{2} \mathrm{O}_{2}$-induced DNA damage in lymphocyte taken from healthy individuals and pre-cancerous patients. Figure shows four groups of treatments including the negative control, excipient mixture, positive control, MYR B $(10 \mu \mathrm{M})$ co-supplemented with $\mathrm{H}_{2} \mathrm{O}_{2}$ and MYR N $(20 \mu \mathrm{M})$ co-supplemented with $\mathrm{H}_{2} \mathrm{O}_{2}$. All treatments were compared against the PC. The mean control values for the \% tail DNA of NC and PC group for healthy individuals and patients were 9, 22 and 18, 29 , respectively, measuring 100 cells each per experiment. Asterisk shows significant difference between the groups. The horizontal lines on top of the graph show the significance difference the positive control and the treatment groups. (For healthy groups $* * P<0.009$, $* * * P<0.005$. For patient groups $* * * * P<0.001, * * * P<0.003)$. b Mean OTM showing the effect of MYR B \& N on $\mathrm{H}_{2} \mathrm{O}_{2}$-induced DNA damage in lymphocytes from healthy volunteers and pre-cancerous patients. Figure shows four groups of treatments including the negative control, positive control, MYR B $(10 \mu \mathrm{M})$ co-supplemented with $\mathrm{H}_{2} \mathrm{O}_{2}$ and MYR N $(20 \mu \mathrm{M})$ co-supplemented with $\mathrm{H}_{2} \mathrm{O}_{2}$. All treatment groups were compared to the PC group. The mean $\mathrm{NC}$ values for the OTM of healthy and patient groups were 0.9 and 4.5 , respectively. The mean maximum values of the PC of healthy and patient groups were 5 and 8 , respectively. The horizontal lines on top of the graph show the significance difference between the positive control and the treatment groups. $(* * * P<0.001$, $* * * P<0.007$ shown on healthy groups and $* * * P<0.002, * * * P<0.001$ for patients groups)
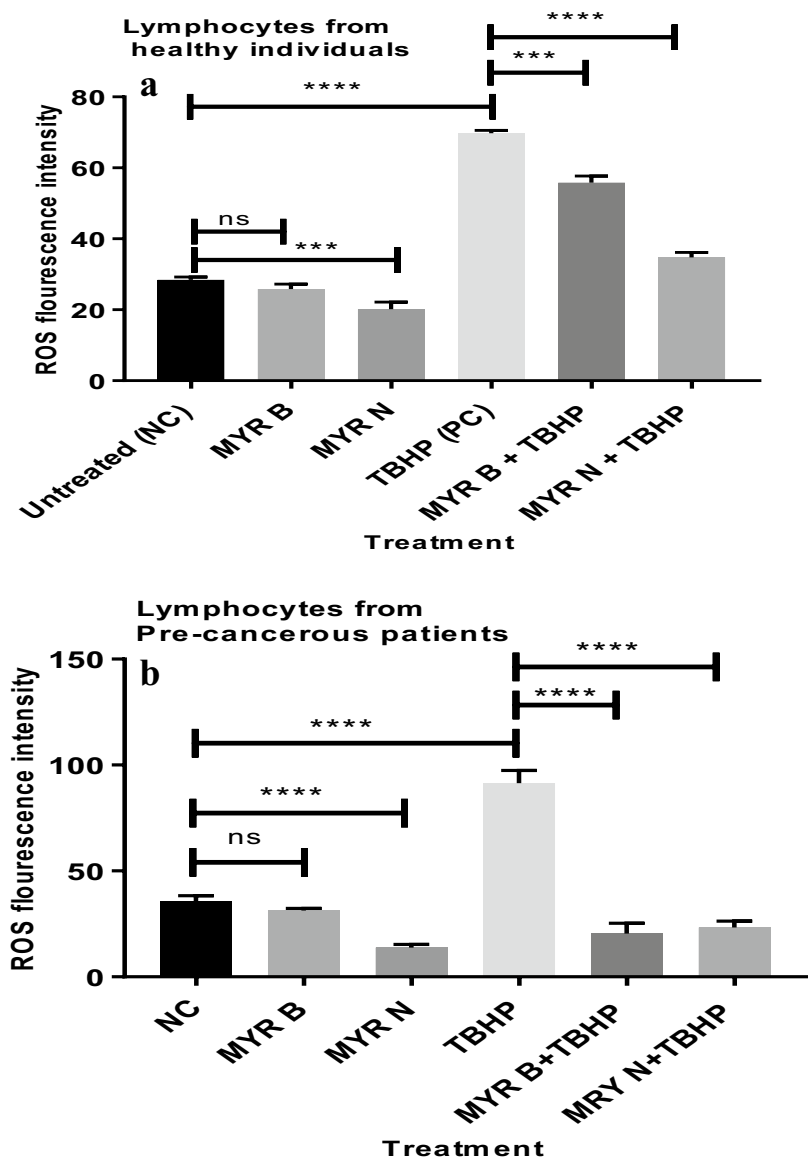

Fig. 4 a Average of 3 independent experiments showing, change in intracellular ROS before and after treatment with TBHP in healthy lymphocyte. The horizontal lines show the significant difference between the groups. $* * * P<0.001, n s$ not significant. Six treatment groups included an untreated group (NC), MYR B $(10 \mu \mathrm{M})$, MYR N $(20 \mu \mathrm{M})$, TBHP $((300 \mu \mathrm{M})$ as PC, TBHP+MYR B, and TBHP + MYR N. b Average of 3 independent experiments showing, change in intracellular ROS before and after treatment with TBHP in lymphocyte from pre-cancerous patients. The horizontal lines show the significant difference between the groups. Ns not significant, $* * * P<0.001$. Six treatment groups included an untreated group (NC), MYR B $(10 \mu \mathrm{M})$, MYR N $(20 \mu \mathrm{M})$, TBHP $((300 \mu \mathrm{M})$ as PC, TBHP+MYR B, and TBHP + MYR N

on GSH levels in lymphocytes from healthy individuals and in those from pre-cancerous patients (Fig. 5a, b).

\section{Effect of myricetin on DSB formation in lymphocytes at basal levels}

DSBs in the nuclei of lymphocyte cells were immunocytochemically stained using the $\gamma \mathrm{H} 2 \mathrm{AX}$ protein, and $\gamma \mathrm{H} 2 \mathrm{AX}$ foci inside each nucleus were counted in 100 cells each. One DSB represented one $\gamma \mathrm{H} 2 \mathrm{AX}$ focus. Results showed that myricetin does not cause DSBs in healthy lymphocytes (Fig. 6a, b). Compared to the number of foci generated in the control, both forms of myricetin (MYR B and 

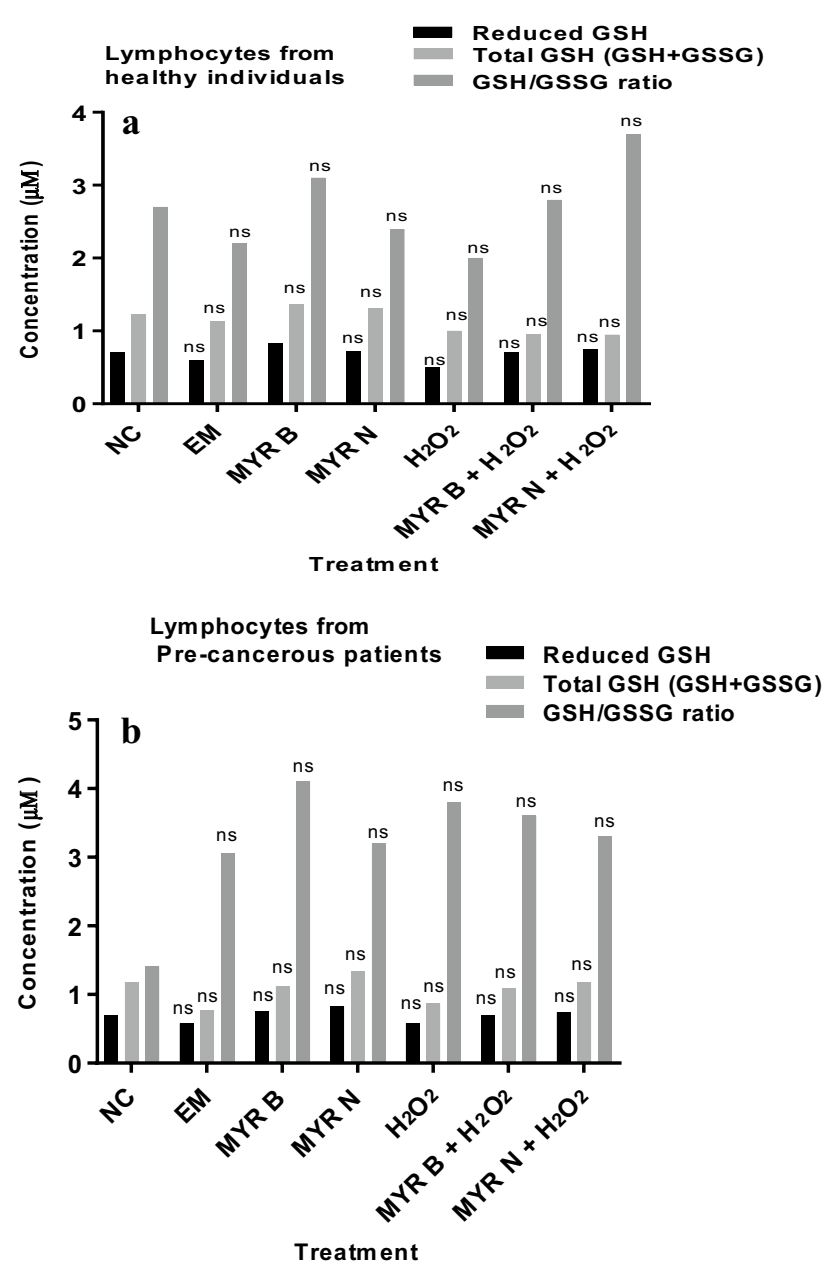

Fig. 5 Levels of different forms of GSH in healthy lymphocyte (a) and those from pre-cancerous patients (b). Cells lysed to the concentration of $1 \times 10^{5}$ cells $/ \mathrm{ml}$. Various treatment groups included the NC (untreated), EX, MYR B $(10 \mu \mathrm{M})$, MYR N $(20 \mu \mathrm{M}), \mathrm{H}_{2} \mathrm{O}_{2}(50 \mu \mathrm{M})$ as $\mathrm{PC}$, MYR B $+\mathrm{H}_{2} \mathrm{O}_{2}$, and MYR N $+\mathrm{H}_{2} \mathrm{O}_{2}$. ns not significant

MYR N) have not shown any significant increase in foci formation. However, as shown in Fig. 6b, an increased number of $\gamma \mathrm{H} 2 \mathrm{AX}$ foci formation was observed after treatment of the healthy lymphocytes with doxorubicin, a known strand break inducer (PC) $(p<0.001)$. Foci formation was observed at basal levels in untreated group from pre-cancerous patients and this incidence of the foci formation was significantly increased $(p<0.001)$ after doxorubicin treatments. Upon treatment with MYR B $(10 \mu \mathrm{M})$ and MYR N $(20 \mu \mathrm{M})$ forms in lymphocytes from pre-cancerous patients, no significant effect was observed in $\gamma \mathrm{H} 2 \mathrm{AX}$ foci formation compared to the untreated group. This suggests that myricetin does not induce DSBs in healthy lymphocytes at basal levels and could potentially provide protection against DSBs formation in lymphocytes from pre-cancerous patients at basal levels.

\section{Discussion}

In this study, we determined the effects of myricetin against $\mathrm{H}_{2} \mathrm{O}_{2}$-induced DNA damage by simultaneously exposing the cells to $\mathrm{H}_{2} \mathrm{O}_{2}(50 \mu \mathrm{M})$ in the presence or absence of MYR B or MYR N. The Comet assay results have shown the protective effect of myricetin bulk and nanoparticles (NPs) against $\mathrm{H}_{2} \mathrm{O}_{2}$-induced ROS-related oxidative damage in the lymphocytes of healthy individuals and pre-cancerous MGUS patients. The basal DNA damage was significantly inhibited when compared to the positive control (Fig. 3a, b). Both forms of myricetin have shown inhibitory effects, whereas MYR N was more effective in causing the reduction in DNA damage, may be due to its enhanced physio-chemical characteristics $(p<0.001)$. The potential explanation for this could be that NPs due to their minute size can easily reach the nucleus through diffusion across the nuclear membrane or transportation via the nuclear pores and gain direct interaction with the DNA (Magdolenova et al. 2012, 2014). This could be further investigated and confirmed by studying the subcellular distribution of these particles using transmission electron microscopy (TEM).

ROS play a crucial role in stimulating DNA strand breaks formation and causing oxidative stress (Tanaka et al. 2007). Accumulation of the DNA damage induced by ROS can lead to various deleterious processes including the stimulation of neoplasms production and eventually tumour development (Tanaka et al. 2007; Vilenchik and Knudson 2003). To evaluate the antioxidant potential of myricetin, we investigated its antioxidant effects in lymphocytes from healthy individuals and pre-cancerous MGUS patients by measuring intracellular ROS contents. The basal levels of ROS and the effect of myricetin against TBHP-induced ROS were evaluated. Results have demonstrated that myricetin has reduced the basal damage in lymphocytes from both the investigated groups as well as the insults introduced by TBHP treatment (Fig. 4a, b). Both forms of myricetin have shown strong antioxidant defence by scavenging the free radicals caused by ROS and by reducing their levels intracellularly. However, when two forms were compared, MYR N $(20 \mu \mathrm{M})$ demonstrated more protective effects than MYR B $(10 \mu \mathrm{M})$. Our results are consistent with previous studies (Wang et al. 2010; Kang et al. 2010). Inhibition of ROS may prevent its accumulation, also the interaction of floating radicals with cellular contents and DNA fragmentation, avoiding DNA lesions and strand breaks. Hence, myricetin shows anti-mutagenic and anti-carcinogenic properties by preventing mutations at non-genotoxic concentrations used for both forms of myricetin. These results are consistent with previous studies conducted on various cells lines 
a
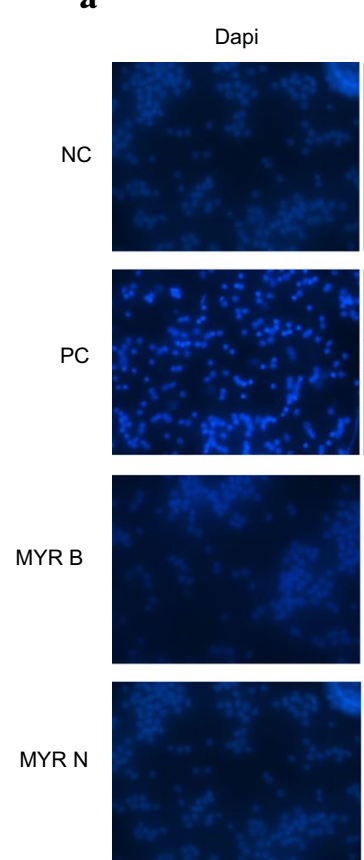

Control lymphocytes

$$
\mathrm{Y}-\mathrm{H} 2 \mathrm{AX}
$$
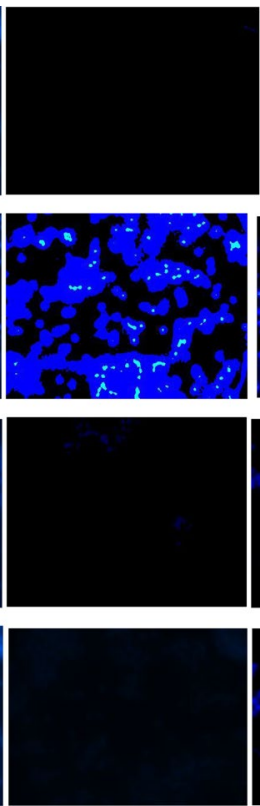
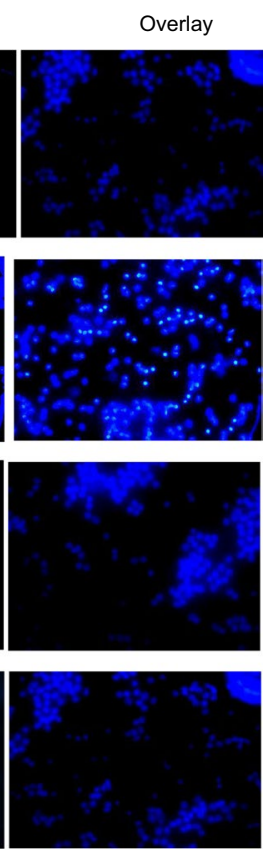

NC

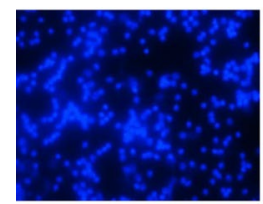

PC
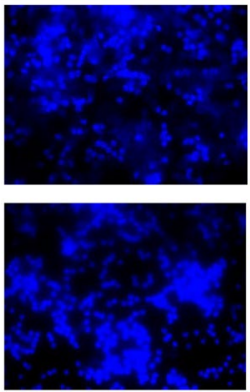

MYR N

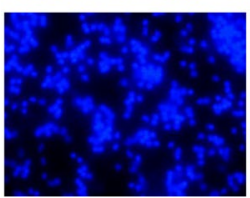

Pre-cancerous Patients' lymphocytes

$\mathrm{Y}-\mathrm{H} 2 \mathrm{AX}$

Overlay
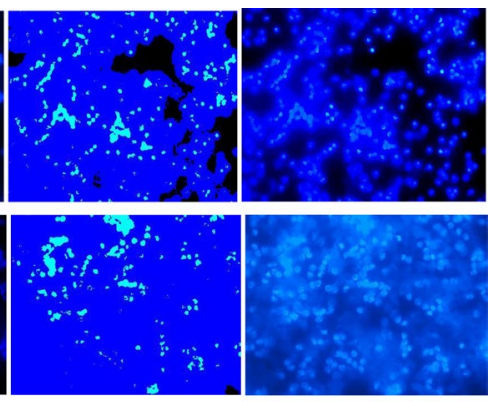

MYR B
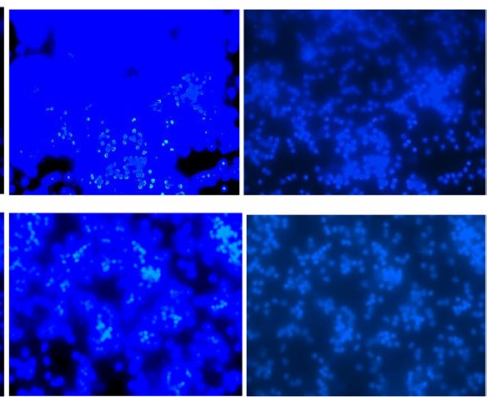

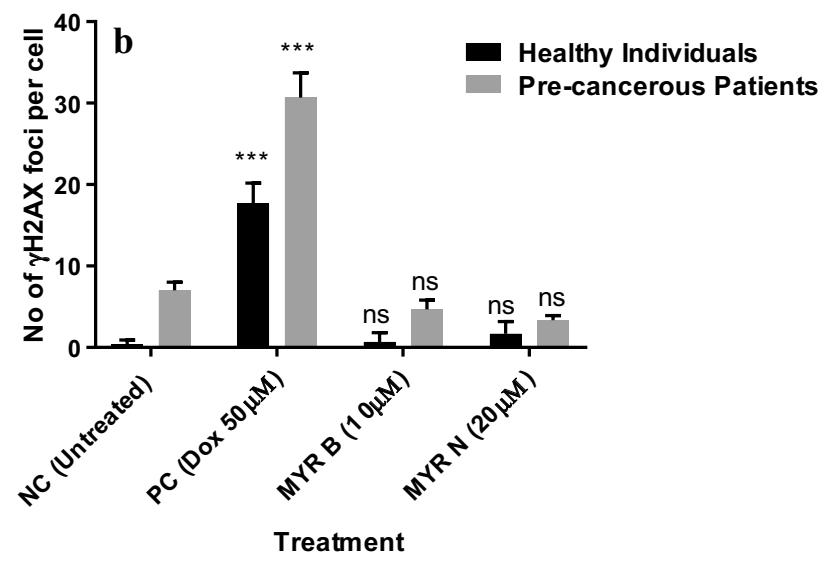

Fig. 6 a $\gamma \mathrm{H} 2 \mathrm{AX}$ phosphorylation in lymphocytes from healthy individuals and pre-cancerous patients presented in untreated cells (NC) and treated with doxorubicin $(50 \mu \mathrm{M})$, MYR B $(10 \mathrm{Mm})$, and MYR $\mathrm{N}(20 \mu \mathrm{M})$. a Merged Dapi and gamma-H2AX stains in blood lymphocytes from healthy individuals and pre-cancerous patients. Num-

using myricetin. It has been previously shown that myricetin protects cells from $\mathrm{H}_{2} \mathrm{O}_{2}$ damage by inhibiting ROS production and by stimulating the antioxidant enzymes. It restored the function of antioxidant defence enzymes such as catalase (CAT), superoxide dismutase (SOD), and glutathione peroxidase (GPx) which was reduced by $\mathrm{H}_{2} \mathrm{O}_{2}$ treatment (Wang et al. 2010).

Although we used the fluorescent probe DCFDA to detect ROS levels, the formamido pyrimidine glycosylase (FPG) protein could also be considered to assess oxidative DNA damage using the Comet assay (Müller et al. 2013). ber of phosphorylated H2AX-foci corresponds with DSBs. b $\gamma \mathrm{H} 2 \mathrm{AX}$ foci induction in lymphocytes with different treatment groups. Data were analysed by two-way ANOVA followed by multiple comparison test for significant differences compared to the untreated control for each group $(* * * p<0.001, n s$ not significant)

Furthermore, this study also investigated the effect of myricetin on intracellular glutathione levels. In healthy cells, the total glutathione pool is mostly in the reduced form (GSH), a key tissue antioxidant that presents first line of defense against ROS. However, when cells are exposed to increased levels of oxidative stress, oxidized glutathione (GSSG) starts gathering and the ratio of GSSG to GSH rises. Hence, a bigger ratio of GSSG-to-GSH is an indication of oxidative stress (Roy and Sil 2012). Our results have demonstrated no significant effect on GSH levels 
after treatment with MYR B and MYR N in lymphocytes from healthy and MGUS patient groups (Fig. 5a, b).

Gamma-H2AX is used as an effective biomarker of DSBs. An accumulating body of evidence suggests that the crucial role of $\gamma \mathrm{H} 2 \mathrm{AX}$ phosphorylation for nuclear foci formation at DBS sites and stimulation of DNA repair (Rogakou et al. 1998; Podhorecka et al. 2010). There exists a one-to-one correspondence between DSBs and gammaH2AX foci; hence, DSBs can be easily visualised immunocytochemically (Rogakou et al. 1999). The number of foci, therefore, could be used as a relative parameter to estimate DNA damage and repair. To evaluate the effect of myricetin on DSBs formation in lymphocytes from healthy individuals and those from pre-cancerous MGUS patients, we quantified $\gamma \mathrm{H} 2 \mathrm{AX}$ foci intensity using immunofluorescence. Our results have shown that myricetin does not induce DSBs formation in healthy lymphocyte and those from the pre-cancerous patients at basal levels (Fig. 6a, b) which is consistent with our previous results from the Comet assay where no significant DNA damage was caused upon exposure to MYR B $(10 \mu \mathrm{M})$ and MYR $\mathrm{N}(20 \mu \mathrm{M})$. However, a trend to lower an overall intensity of $\gamma \mathrm{H} 2 \mathrm{AX}$ foci was observed in the lymphocytes from precancerous MGUS patients treated with MYR B and MYR $\mathrm{N}$ when compared to the untreated group. This marks the current study as the first one to demonstrate the effect of MYR B $(10 \mu \mathrm{M})$ and MYR N $(20 \mu \mathrm{M})$ on DSBs development in lymphocytes from healthy individuals and those from pre-cancerous MGUS patients at basal levels.

In conclusion, this work demonstrates for the first time (to our knowledge) that myricetin bulk and nano at selective non-genotoxic concentrations protect the lymphocytes (from healthy individuals and pre-cancerous patients) from damaging effects of TBHP and $\mathrm{H}_{2} \mathrm{O}_{2}$ primarily by inhibiting ROS-induced oxidative stress. Besides this, MYR B $(10 \mu \mathrm{M})$ and MYR N $(20 \mu \mathrm{M})$ do not induce formation of DSBs in lymphocytes from healthy individuals and pre-cancerous MGUS patients at basal levels and could possibly protect the lymphocytes from extensive cell damage by inhibiting DSBs formation and ultimately help in cell survival and DNA damage repair. DNA repair capacity of lymphocytes after treatment with myricetin could possibly be studied using the Comet assay simply by assessing the damage at different time periods (Collins 2004). The overall results have demonstrated that MYR N $(20 \mu \mathrm{M})$ has shown better antioxidant and genoprotective effects against the oxidative damage in lymphocytes from healthy individuals and pre-cancerous MGUS patients when compared to MYR B $(10 \mu \mathrm{M})$.

Acknowledgements This study was funded by Mr Nasir Qayyum, Bradford, UK. Blood samples from patient group were kindly provided by Jan Bunch and Victoria Drew, headed by Dr Lisa Newton, Bradford Royal Infirmary (BRI), Department of Haematology, Bradford, UK.

\section{Compliance with ethical standards}

Conflict of interest Authors declare no conflict of interest.

Open Access This article is licensed under a Creative Commons Attribution 4.0 International License, which permits use, sharing, adaptation, distribution and reproduction in any medium or format, as long as you give appropriate credit to the original author(s) and the source, provide a link to the Creative Commons licence, and indicate if changes were made. The images or other third party material in this article are included in the article's Creative Commons licence, unless indicated otherwise in a credit line to the material. If material is not included in the article's Creative Commons licence and your intended use is not permitted by statutory regulation or exceeds the permitted use, you will need to obtain permission directly from the copyright holder. To view a copy of this licence, visit http://creativecommons.org/licenses/by/4.0/.

\section{References}

Anderson D et al (2014) Sensitivity and specificity of the empirical lymphocyte genome sensitivity (LGS) assay: implications for improving cancer diagnostics. FASEB J 28(10):4563-4570

Azqueta A, Dusinska M (2015) The use of the comet assay for the evaluation of the genotoxicity of nanomaterials. Front Genet 6:239

Benatti U, Morelli A, Damiani G, De Flora A (1982) A methemoglobin dependent and plasma-stimulated experimental model of oxidative hemolysis. Biochem Biophys Res Commun 106:183-1190

Bhattacharya S, Gachhui R, Sil PC (2011) Hepato-protective properties of Kombucha tea against TBHP-induced oxidative stress via suppression of mitochondria dependent apoptosis. Pathophysiology 18:221-234

Burdick AD et al (2003) Benzo(\%3cstrong\%3e $\% 3$ cem $\% 3 \mathrm{ea} \% 3 \mathrm{c} /$ em $\% 3 \mathrm{e} \% 3 \mathrm{c} /$ strong $\% 3 \mathrm{e})$ pyrene quinones increase cell proliferation, generate reactive oxygen species, and transactivate the epidermal growth factor receptor in breast epithelial cells. Cancer Res 63(22):7825-7833

Chao-Wei Chen K et al (2004) Platelet derived growth factor (PDGF)induced reactive oxygen species in the lens epithelial cells: the redox signaling. Exp Eye Res 78(6):1057-1067

Chen W, Li Y, Li J, Han Q, Ye L, Li A (2011) Myricetin affords protection against peroxynitrite-mediated DNA damage and hydroxyl radical formation. Food Chem Toxicol 49:2439-2444

Chobot V, Hadacek F (2011) Exploration of pro-oxidant and antioxidant activities of the flavonoid myricetin. Redox Rep 16:242-247

Collins AR (2004) The comet assay for DNA damage and repair: principles, applications, and limitations. Mol Biotechnol 26(3):249-261

Deuticke B, Heller KB, Haest WM (1987) Progressive oxidative membrane damage in erythrocytes after pulse treatment with tbutylhydroperoxide. Biochimica et Biophysica Acta 899:113-124

Dhodapkar MV (2016) MGUS to myeloma: a mysterious gammopathy of underexplored significance. Blood 128(23):2599-2606

Duthie SJ et al (1997) Quercetin and myricetin protect against hydrogen peroxide-induced DNA damage (strand breaks and oxidised pyrimidines) in human lymphocytes. Mutat Res Genet Toxicol Environ Mutagenesis 393(3):223-231

Fraga CG, Martino VS, Ferraro GE, Coussio JD, Boveris A (1987) Flavonoids as antioxidants evaluated by in vitro and in situ liver chemiluminescence. Biochem Pharmacol 36:717-720

Kang KA et al (2010) Myricetin protects cells against oxidative stressinduced apoptosis via regulation of PI3K/Akt and MAPK signaling pathways. Int J Mol Sci 11(11):4348-4360 
Laabich A et al (2007) Protective effects of myricetin and related flavonols against $\mathrm{A} 2 \mathrm{E}$ and light mediated-cell death in bovine retinal primary cell culture. Exp Eye Res 85(1):154-165

Laubenthal J et al (2012) Cigarette smoke-induced transgenerational alterations in genome stability in cord blood of human F1 offspring. FASEB J 26(10):3946-3956

Magdolenova $\mathrm{Z}$ et al (2012) Can standard genotoxicity tests be applied to nanoparticles? J Toxicol Environ Health Part A 75(13-15):800-8061

Magdolenova Z et al (2014) Mechanisms of genotoxicity. A review of in vitro and in vivo studies with engineered nanoparticles. Nanotoxicology 8(3):233-278

Müller N, Moreno-Villanueva M, Fischbach A, Kienhöfer J, Martello R, Dedon PC, Ullrich V, Bürkle A, Mangerich A (2013) An automated Fpg-based FADU method for the detection of oxidative DNA lesions and screening of antioxidants. Toxicoloy 310:15-21

OECD (2016) Test No. 489: in vivo mammalian alkaline comet assay. OECD Publishing, Paris

Pandey KB, Mishra N, Rizvi SI (2009) Myricetin may provide protection against oxidative stress in type 2 diabetic erythrocytes. $\mathrm{Z}$ Naturforsch C 64:626-630

Peng IW, Kuo SM (2003) Research communication: flavonoid structure affects the inhibition of lipid peroxidation in Caco-2 intestinal cells at physiological concentrations. J Nutr 133(7):2184-2187

Perkins CL, Fang G, Kim CN, Bhalla KN (2000) The role of Apaf-1, caspase- 9, and bid proteins in etoposide- or paclitaxel-induced mitochondrial events during apoptosis. Cancer Res 60:1645-1653

Podhorecka M et al (2010) H2AX phosphorylation: its role in DNA damage response and cancer therapy. J Nucleic Acids. https://doi. org/10.4061/2010/920161

Rogakou EP, Pilch DR, Orr AH, Ivanova BS, Bonner WM (1998) DNA double-stranded breaks induce histone $\mathrm{H} 2 \mathrm{AX}$ phosphorylation on serine 139. J Biol Chem 273:5858-5868

Rogakou EP et al (1999) Megabase chromatin domains involved in DNA double-stranded breaks in vivo. J Cell Biol 146(5):905-916

Roy A, Sil PC (2012) Tertiary butyl hydroperoxide induced oxidative damage in mice erythrocytes: protection by taurine. Pathophysiology 19(2):137-148

Sarkar MK, Sil PC (2010) Prevention of tertiary butyl hydroperoxide induced oxidative impairment and cell death by a novel antioxidant protein molecule isolated from the herb Phyllanthus niruri. Toxicology In Vitro 24:1711-1719
Schmid TE et al (2010) Differences in the kinetics of $\gamma$-H2AX fluorescence decay after exposure to low and high LET radiation. Int J Radiat Biol 86(8):682-691

Semwal DK et al (2016) Myricetin: a dietary molecule with diverse biological activities. Nutrients 8(2):90

Shimmyo Y et al (2008) Multifunction of myricetin on A $\beta$ : neuroprotection via a conformational change of $A \beta$ and reduction of $A \beta$ via the interference of secretases. J Neurosci Res 86(2):368-377

Singh NP et al (1988) A simple technique for quantitation of low levels of DNA damage in individual cells. Exp Cell Res 175(1):184-191

Slikker W, Desai VG, Duhart H, Feuers R, Imam SZ (2001) Hypothermia enhances bcl-2 expression and protects against oxidative stress induced cell death in Chinese hamster ovary cells. Free Radic Biol Med 31:405-411

Tanaka T, Huang X, Halicka HD et al (2007) Cytometry of ATM activation and histone $\mathrm{H} 2 \mathrm{AX}$ phosphorylation to estimate extent of DNA damage induced by exogenous agents. Cytometry Part A 71(9):648-661

Tice RR, Agurell E, Anderson D, Burlinson B, Hartmann A, Kobayashi H, Miyamae Y, Rojas E, Ryu JC, Sasaki YF (2000) Single cell gel/ comet assay: guidelines for in vitro and in vivo genetic toxicology testing. Environ Mol Mutagen 35:206-221

Varbiro G et al (2001) Direct effect of Taxol on free radical formation and mitochondrial permeability transition. Free Rad Biol Med 31(4):548-558

Vilenchik MM, Knudson AG (2003) Endogenous DNA double-strand breaks: production, fidelity of repair, and induction of cancer. Proc Natl Acad Sci USA 100(22):12871-12876

Wang ZH et al (2010) Myricetin suppresses oxidative stress-induced cell damage via both direct and indirect antioxidant action. Environ Toxicol Pharmacol 29(1):12-18

Zavodnik LB, Zavodnik IB, Niekurzak A, Szosland K, Bryszewska M (1988) Activation of red blood cell glutathione peroxidase and morphological transformation of erythrocytes under the action of tert-butyl hydroperoxide. Biochem Mol Biol Int 44:577-588

Publisher's Note Springer Nature remains neutral with regard to jurisdictional claims in published maps and institutional affiliations. 